\title{
EMBEDDED WRIST WATCH
}

\author{
Kishore Deka \\ COMPUTER SCIENCE ENGINEERING, DBCET, GUWAHATI, INDIA, kisksddeka@gmail.com
}

\begin{abstract}
This paper introduces WRIST WATCH WITH EMBEDDED SYSTEMS- a wrist watch having all the functions of a cell phone. The services include- (1). Calling, (2). Video recording, (3). Image capturing, (4). Voice recording, (5). Song recording, (6). Media player, (7). Game, (8). Internet connectivity, (9). Calculator, (10). FM and AM radio connection, (11). Message transmission and receiving. The same wrist watch will also act as medical test equipment. The types of test that can be incorporated are-(1). Blood test, (2). Different types of disease detection and daily control analysis. Collectively the wrist watch can be used as a modern cell phone having all the facilities we can inherit and also it can use as for managing or analyzing the daily common diseases.
\end{abstract}

$* * *$

\section{INTRODUCTION}

Usually, a wrist watch can be used for watching or observing time along with alarm settings or some familiar functions in day-to- day life. But apart from this, it can be used as a cell phone having the facilities of calling, message transmission and receiving, voice and video recording, FM and AM radio, photo capturing. It also has memory card slots along with sim card slots. It can also be used as for playing different types of games. It will also have internet connectivity. Apart from the cell phone extension, the same wrist watch can also be used as medical testing equipment. It can be used for testing diabetes, blood pressure, tuberculosis, Rh factor, RBC count, WBC count, plasma count, hemoglobin count and all the bloodrelated diseases or the blood related queries like the blood group of a person. It also acts as a running pedometer. It can also help in controlling blood pressure. It can also be used as BMI calculator, BMR calculator, body fat calculator, activity calculator, calorie calculator.

\section{LITERATIVE STUDY}

By looking into the various concepts, materials and othealike things or rather records in internet and the books available to us, it has been found where it is clearly stated that already the kinds of wrist watches havin all the functions of the cellphone that we have discussed in the previous section of our discussion has been clearly and successfully incorpated in this real world. The Wrist watch with embedded mobile applications can be categorized into three categories, on the basis of a close observation or study. The three categories are briefly described below and the three categories are-

\section{A. Wrist watch with keypad}

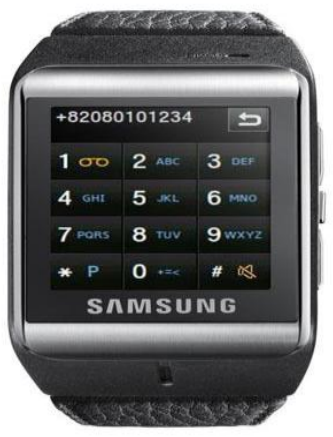

\section{FIG 1: WRIST WATCH WITH KEYPAD}

This is a type of wrist watch with keypad which is having the keypad in the screen itself. This model is little outdated. An image of it is clearly displayed in figure 1. It is unable to display time. Though it appears as a screen touch cell phone but it is not. It has only three functions making a call, receiving a call, message transmission and receiving and setting alarm.

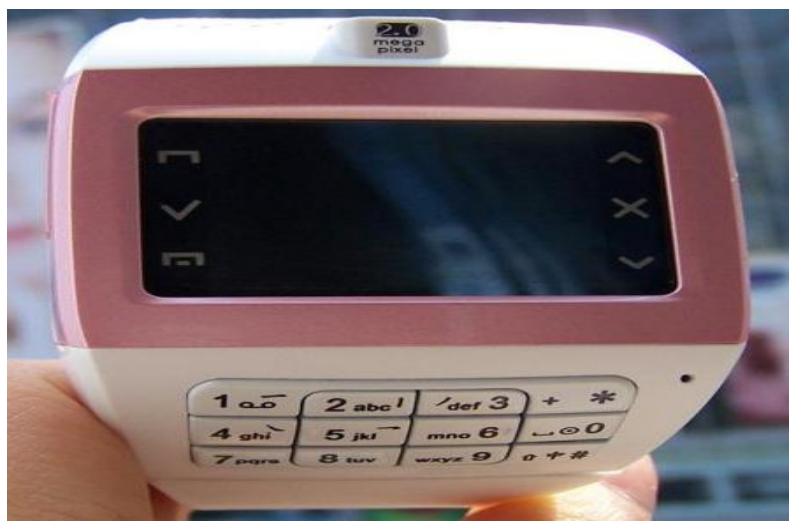

Fig 2: wrist watch with keypad 
The above figure clearly depicts a wrist watch which is having keypad installed in it. Unlikely the previous model described above (figure 1), which is having its keypad in the screen; the new model is having the keypad on the strip (wrist watch belt) of the wrist watch. If we see it clearly one can also depict that it is having a camera with a standard rating of 2 megapixels. It has also a microphone in it which will help to capture the voice of the user and will be helpful in transmitting it.

It is the simplest types of wrist watch having an embedded cell phone in it. It simply has the function of making a call, receiving a call, image capturing, FM radio, playing game, voice recording, timer, calculator, saving notes, memorandum, message transmission and receiving, setting alarm and the alike things. It does not have memory card slots or it have media player or video player. It does not have any wifi connectivity or Bluetooth or infrared connection. It only have image viewer, where we can zoom the image, edit the image and do all kinds of image related operations.

\section{B. Wrist watch having smart applications}

This is a type of wrist watch in which keypads are not present. An image of it is displayed in figure 3. In this type of model, in the strip (belt) of the wrist watch, there is just a call (green button) and a call end (red button). It has a joystick in the side which toggles between the options and the both button in which dot (.) is present (as shown in the figure) near both call and call end buttons gives the option to open, go back read, write message and all the alike things available. The options available will be displayed on the screen and after watching or observing the screen only, one can know what action should be taken or which button out of this two buttons should be pressed. It can also display time (analogue clock) on the wrist watch.

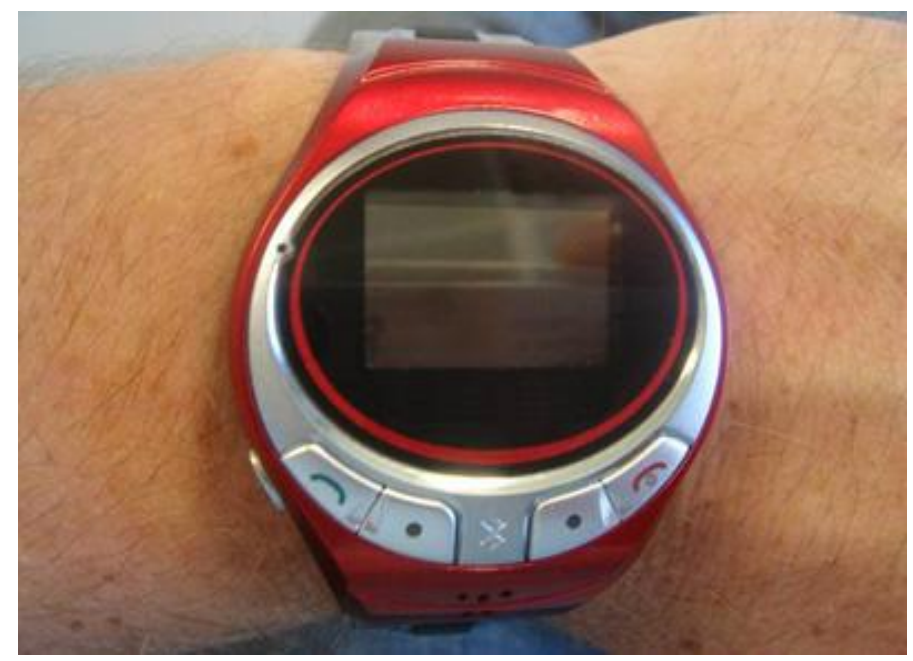

Fig 3: Wrist watch having smart application
It is the type of wrist watch having an embedded cell phone having smart applications in it. It has the function of making a call, receiving a call, playing game, calculator, saving notes, memorandum, message transmission and receiving, FM radio, timer control, image capturing, video recording, voice recording, song recording, setting alarm. It also has packet data connection, Bluetooth and also infrared connection (in some models). Till now only $2 \mathrm{G}$ connection can be incorporated in this type of model, $3 \mathrm{G}$ or $4 \mathrm{G}$ connection is till now, but in the near future, $3 \mathrm{G}$ or $4 \mathrm{G}$ is expected to make an impact in near future. For the concern in terms of economic feasibility, laymen can afford it i.e. it is economically feasible to common people. It has also games which even has java games in some models. It has memory slots (microsd) expandable till $8 \mathrm{~GB}$ (till date). It is also having a sim card slot having the facility of a single sim only.

\section{Wrist watch having android applications}

It is a type of wrist watch in which keypads are also not present. It is purely screen touch with android applications incorporated in it. The image of it is displayed in figure 4. It is having touch digi pen in it. It also does not have any buttons present anywhere on the wrist watch (whether it be on the strips of the wrist watch or in and around the wrist watch). It also can display time (analogue as well as digital clock) on the wrist watch.

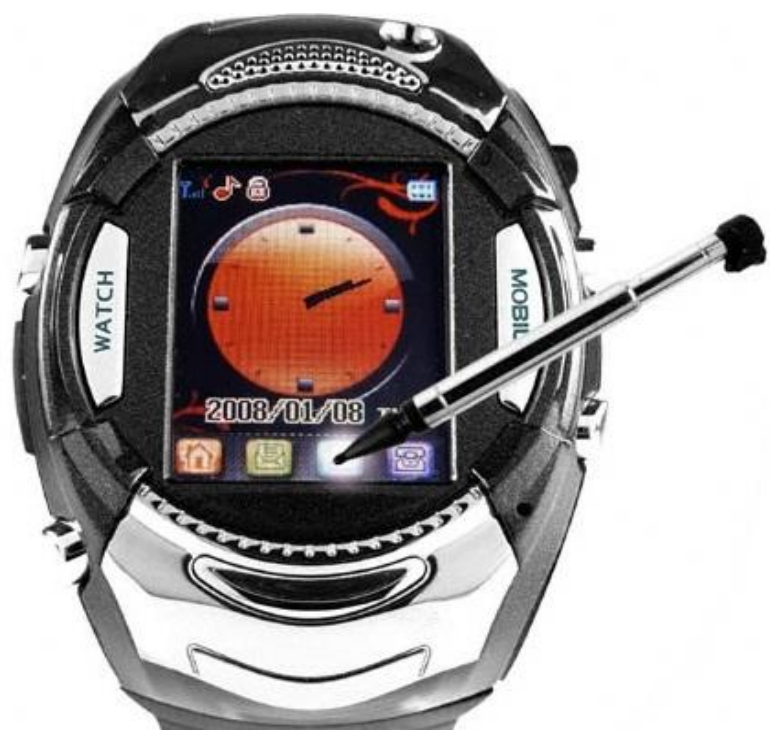

Fig 4: Wrist watch having android application

It is the type of wrist watch having an embedded cell phone having smart applications in it. It has the function of making a call, receiving a call, playing game, calculator, saving notes, memorandum, message transmission and receiving, FM radio, timer control, image capturing, video recording, voice recording, song recording, setting alarm. It also has packet data connection, Bluetooth and also infrared connection. It also has 
wifi connection facility in it. It also has java enabled game loaded in it. It has memory card slot (microSD) expandable up to $32 \mathrm{~GB}$. It also has a memory card slot having the facility of inserting two Sims at the same time (dual sim). It also has GPS connectivity in it in which we can trace where we are at the moment, what are the hotels, entertaining places, cinema halls, shopping malls near or local to that place. It also gives a satellite image of the whole area (terrain). It also has the facility of providing navigation to a place in satellite images. It also has radio connectivity which has both FM and AM facilities. It has file reading options. It can display file in pdf format and also word format. One can edit the document and can also zoom, copy and paste anything on the document. Besides all this, this type of wrist watch also has smart voice detection in which if the user speaks something or rather orders anything, immediately the smart phone will search or do anything to give a required action to the order given by the user.

\section{Comparision between all the three types of wrist watches}

By observing the comparison one could figure it out in which respect which one of is better and according to it one could make a choice which one is more preferable to them in their own terms of consideration.

TABLE 1:Comparison between the different types

\begin{tabular}{llll}
\hline & TYPE A & TYPE B & TYPE C \\
\hline PRICE & LOW & MEDIUM & HIGH \\
USER- & LESS & MEDIUM & MORE \\
FRIENDLY & & & \\
DESIGN & LOW & MEDIUM & HIGH \\
FEATURES & LESS & MEDIUM & MORE \\
BATTERY & GOOD & MEDIUM & MEDIUM \\
MULTIMIEDIA & NO & YES & YES \\
RADIO & NO & FM & FM,AM \\
BLUETOOTH & NO & YES & YES \\
INFRARED & NO & NO & YES \\
WIFI & NO & NO & YES \\
HEADPHONE & NO & YES & YES \\
SPEAKER & NO & NO & YES \\
\hline
\end{tabular}

\section{EXISTING METHODOLOGY}

There is already an existing model which will be described in this section. Along with it, some new concepts will be described or explained briefly so that one can fully understand the new concept or the idea fully.

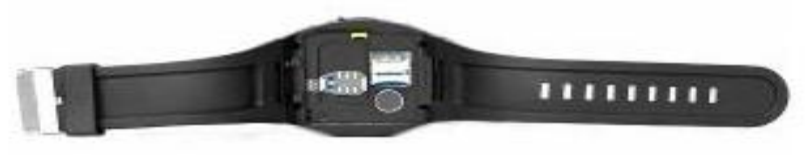

Fig 5: Backside view

The backside view (dissected image) can be seen where the battery (Lithium Ion) has been removed. As a result, memory card slots along with the memory card slots. In figure 5 , which will display a more magnified detailed image of the backside view the battery along with the type of memory card inserted in it is clearly shown.

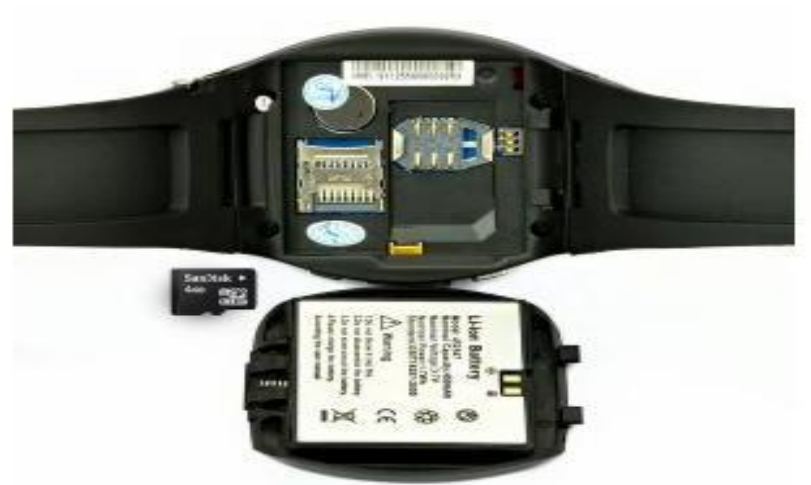

Fig 6: Backside view

The wrist watch can also be connected to a laptop/computer via Bluetooth connection as well as through usb connectivity. It also has a power button to switch it on or off (figure 7).

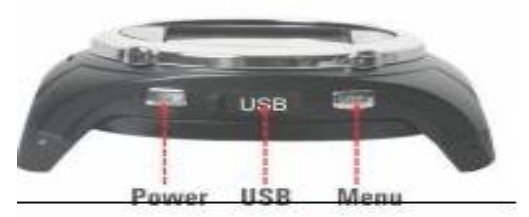

Fig 7: Side view

The menu button is also clearly visible in which the menu opens up in the screen and the user can do the required operations that the user wishes to do it. 
Also there is a camera which can be clearly visible in the figure 8. In the existing model, only the camera can capture photos, videos and display it in the image viewer and the video player etc. A wrist watch with the image present in the image viewer can look like as shown clearly on the figure 9.

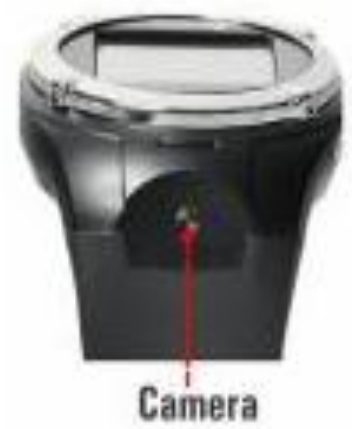

Fig 8: Camera in wrist watch

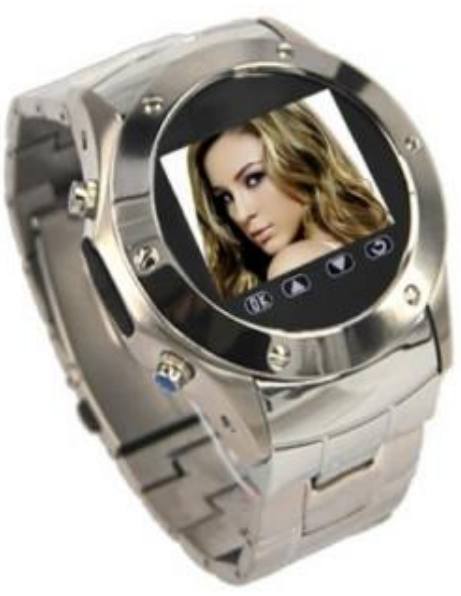

Fig 9: Image in image viewer

There is a common doubt arising in everyone's mind as to how to insert the sim card and the memory card in the sim card slots and the memory card slots respectively. The sim card is the normal sim card commonly used in our day-to-day life It would be rather clear or clarified be in the Figure 10, where it is shown that when and where and how the sim card is inserted. In the same way, the memory card is inserted in the memory card slot on the opposite side of the side where the sim card was been inserted.

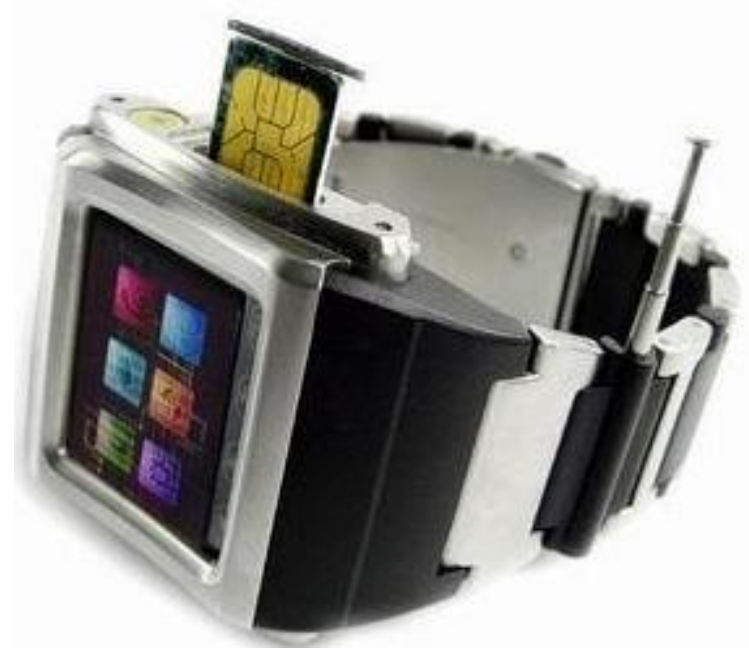

Fig 10: Sim card and its slot

\section{PROPOSED METHODOLOGY}

In Figure 8, the camera is visible; it can be further used as a projector. The LED that is present in the camera can emit the light of an entire document on a nearby surface and as it can emit the document; obviously the image will be magnified. Thus, it will help any person to refer the document on-the-go. In this way, it could act as a great help to mankind as human eye could also don't have to concentrate heavily on the document.

Coming to the medicinal and health concept, in the strip (as in Figure 11) of the wrist watch, there can be small test tubes (ignition tube as in Figure 12) attached in the strip of the wrist watch. The wrist watch strip attached in hand will have a small or needle attached with it, which is inside the wrist watch, which when given the option the needle gets out and pierces a little in the body and the blood can then be transferred to a test tube. In this way, the blood could be collected. This sample so collected on the ignition tubes can be extracted later on and as such it could be further send to any laboratory or blood diagnostic centre by which blood group, Rh factor, RBC count, WBC count, Plasma count etc. and all the blood related queries and the diagnostics can be performed.

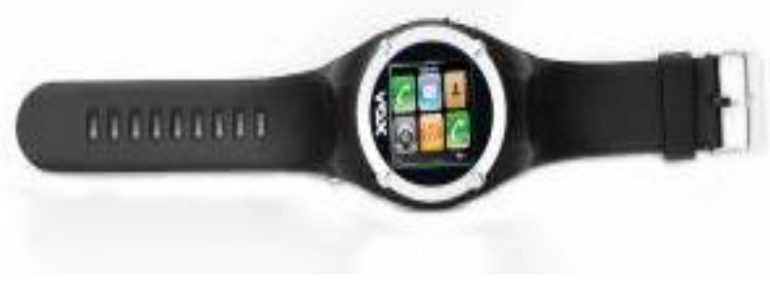

Fig 11: Strip 


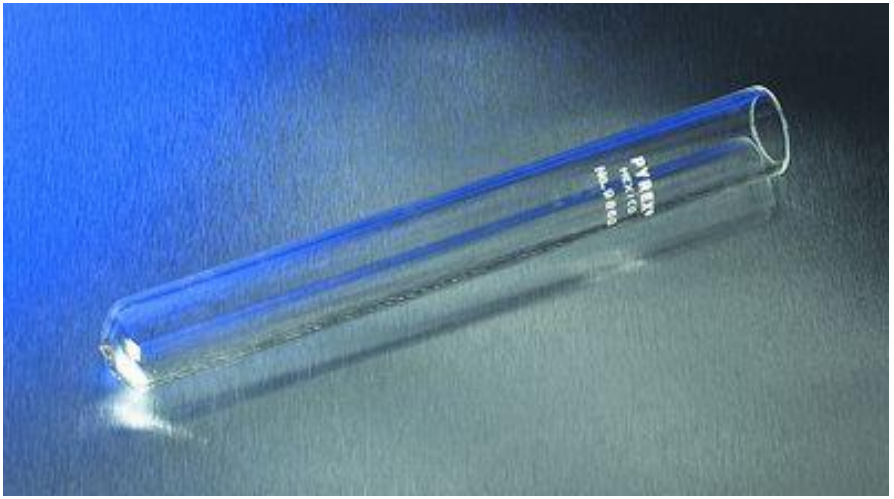

Fig 12: Ignition tube

It can also act as pedometer. The wrist watch has an application through which it regularly checks the amount of sweat that is in touch of the strip and it can calculate and make a conclusion what is the amount of time that the user or the person should walk or run. The same application can be used to control blood pressure. If there is extraordinary sweat emerging in extraordinary time of the day, then a vibration will be encountered, which will not stop automatically. It will alert the user or the person that something regarding the Blood Pressure is wrong and that person can act accordingly in the required appropriate time.

The wrist watch can also be used as for analyzing the diabetes or the sugar level. It can use the concept of "Accu-chek glucometer" for monitoring the level of sugar or the diabetes level. The original Accu-chek is generally large in shape, so accu-chek cannot be directly used here since, it will not be feasible with it. So, the software used inside the device Accuchek is used. The figure of the device accu-chek is in Figure 13.

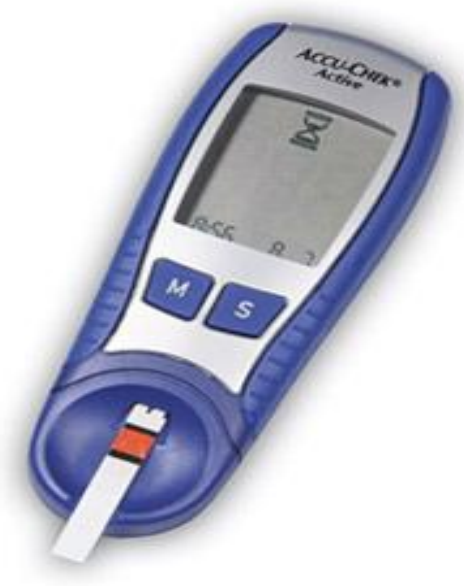

It can also be used for many health-related queries. It can be used for calculating Body Mass Index (BMI). The height and weight of the person is asked. There is fields in which a person can enter and by clicking on calculate the software will be able to calculate. A format of BMI calculator is in Figure 14.

\begin{tabular}{|l|r|}
\hline \multicolumn{2}{|c|}{ Provide your response in the input fields to calculate } \\
\hline Height & $30 \mathrm{~cm} / 0 \mathrm{ft} 11.8 \mathrm{in}$ \\
\hline Calculate & Reset \\
\hline
\end{tabular}

Fig 14: BMI calculator

It can be also used for calculating Basal Metabolic Rate (BMR). The required fields to be entered are both different for male and female since the body requirements are different for both male and female. BMR calculator for both the types are in Figure 15.

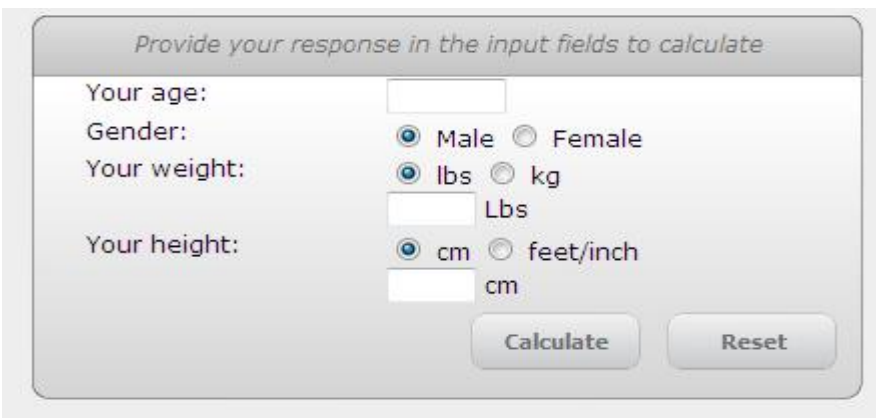

Fig 15: BMR calculator

It can be also used for calculating Body Fat Calculator. The required fields to be entered are both different for male and female since the body requirements are different for both male and female. Body fat calculator for both the types is in Figure 16 and Figure 17.

Provide your response in the input fields to calculate

Female Male $\bigcirc$

Weight

Waist circumference

Wrist circumference

Hip circumference

Forearm circumference

\begin{tabular}{|l}
\hline $\mathrm{kg}^{*}$ \\
\hline $51 \mathrm{~cm} / 20.08 \mathrm{in}=$ \\
\hline $3 \mathrm{~cm} / 1.18 \mathrm{in}=$ \\
$51 \mathrm{~cm} / 20.08 \mathrm{in}=$ \\
$3 \mathrm{~cm} / 1.18 \mathrm{in}=$ \\
Calculate
\end{tabular}

Fig 13: Accu-chek

Fig 16: Body fat calculator (for female) 


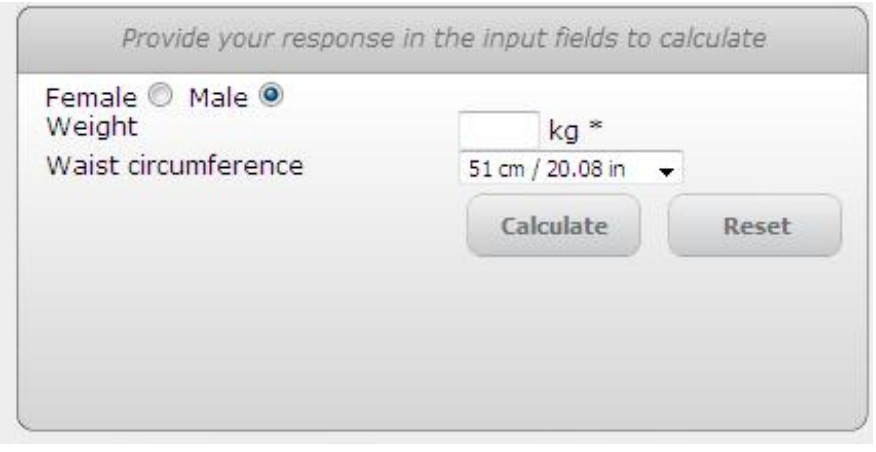

Fig 17: Body fat calculator (for male)

It can be used for calculating activity calculator. There is fields in which a person can enter and by clicking on calculate the software will be able to calculate. A format of activity calculator is in Figure 18.

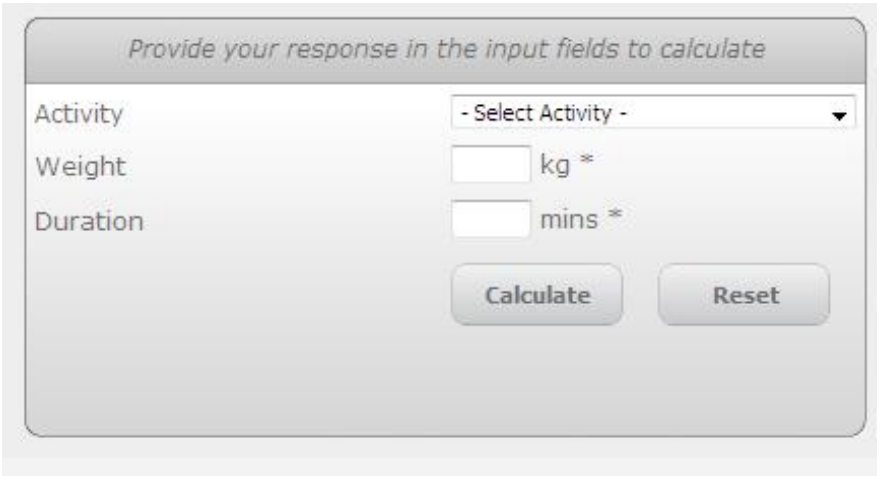

Fig 18: Activity calculator

It can be used for calculating calorie. There are fields in which a person can enter and by clicking on calculate the software will be able to calculate. A format of calorie calculator is in Figure 19.

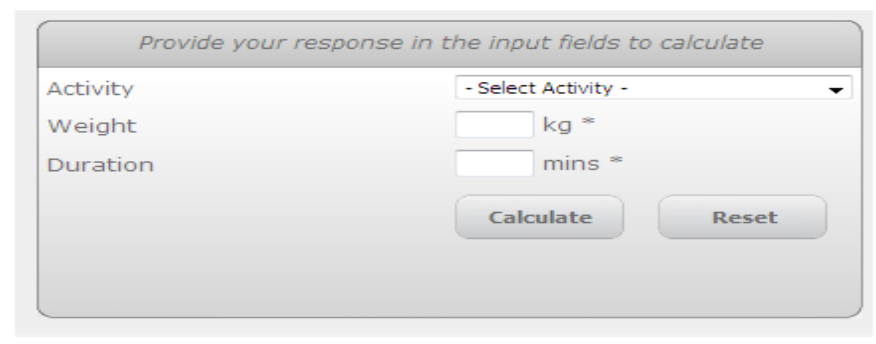

Fig 19: Calorie calculator

\section{CONCLUSIONS}

So, in this discussion we can conclude by saying that wrist watch can be used as a mobile with many features (with almost all the functions). Furthers the concepts or the ideas that can be incorporated on the wrist watch can be extended and can be implemented keeping in consideration the view of economic and technical feasibility.

\section{REFERENCES}

[1] http://www.britishnutritions.in/calorie-calculator.html.

[2] http://www.britishnutritions.in/activity-calculator.html

[3] http://www.britishnutritions.in/bmi-calculator.html

[4] http://www.britishnutritions.in/body-fat-calculator.html

[5] http://www.britishnutritions.in/bmr-calculator.html

[6] http://www.naaptol.com/other-miscellaneousaccessories/liveview-android-

watch/P/12187593.html?ntpromoid=5896\&utm_source =Facebook_Ads\&utm_medium=ADs\&utm_campaign= sonylive_MT\&utm_code=Facebook

[7] http://www.naaptol.com/mobile-handsets/xelectronm998-watch-cum-mobile-phone/P/12142416.html

[8] www.mobilewatch.com 